\title{
Communication \\ High-Yield Production of Nano-Lateral Size Graphene Oxide by High-Power Ultrasonication
}

\author{
Licínia Timochenco ${ }^{1}$, Raquel Costa-Almeida ${ }^{2,3}{ }^{(0)}$, Diana Bogas ${ }^{1}$, Filipa A. L. S. Silva ${ }^{2,3}$, Joana Silva ${ }^{4,5}{ }^{(D}$, \\ André Pereira ${ }^{4,5}$, Fernão D. Magalhães ${ }^{1}$ (D) and Artur M. Pinto $1,2,3, * \mathbb{D}$ \\ 1 LEPABE, Faculdade de Engenharia, Universidade do Porto, 4200-180 Porto, Portugal; \\ up201809122@fe.up.pt (L.T.); dianabogas@gmail.com (D.B.); fdmagalh@fe.up.pt (F.D.M.) \\ 2 i3S-Instituto de Investigação e Inovação em Saúde, Universidade do Porto, 4200-180 Porto, Portugal; \\ rcalmeida@i3s.up.pt (R.C.-A.); flsilva@i3s.up.pt (F.A.L.S.S.) \\ 3 INEB-Instituto de Engenharia Biomédica, Universidade do Porto, Rua Alfredo Allen, 208, \\ 4200-180 Porto, Portugal \\ 4 IFIMUP and IN-Institute of Nanoscience and Nanotechnology, Departamento de Física e Astronomia da \\ Faculdade de Ciências da Universidade do Porto, 4169-007 Porto, Portugal; asilva.joana@gmail.com (J.S.); \\ ampereira@fc.up.pt (A.P.) \\ 5 CFP, Department of Physics Engineering, FEUP, Rua Dr. Roberto Frias, 4200-465 Porto, Portugal \\ * Correspondence: arturp@fe.up.pt
}

\section{check for}

updates

Citation: Timochenco, L.; Costa-Almeida, R.; Bogas, D.; Silva,

F.A.L.S.; Silva, J.; Pereira, A.;

Magalhães, F.D.; Pinto, A.M.

High-Yield Production of Nano-

Lateral Size Graphene Oxide by

High-Power Ultrasonication.

Materials 2021, 14, 1916. https://

doi.org/10.3390/ma14081916

Academic Editor:

Agnieszka Jastrzębska

Received: 13 March 2021

Accepted: 9 April 2021

Published: 12 April 2021

Publisher's Note: MDPI stays neutral with regard to jurisdictional claims in published maps and institutional affiliations.

Copyright: (C) 2021 by the authors. Licensee MDPI, Basel, Switzerland. This article is an open access article distributed under the terms and conditions of the Creative Commons Attribution (CC BY) license (https:// creativecommons.org/licenses/by/ $4.0 /)$.

\begin{abstract}
Nanographene oxide (GOn) constitutes a nanomaterial of high value in the biomedical field. However, large scale production of highly stable aqueous dispersions of GOn is yet to be achieved. In this work, we explored high-power ultrasonication as a method to reduce particle size of GO and characterized the impact of the process on the physicochemical properties of the material. GOn was obtained with lateral dimensions of $99 \pm 43 \mathrm{~nm}$ and surface charge of $-39.9 \pm 2.2 \mathrm{mV}$. High-power ultrasonication enabled an improvement of stability features, particularly by resulting in a decrease of the average particle size, as well as zeta potential, in comparison to GO obtained by lowpower exfoliation and centrifugation $(287 \pm 139 \mathrm{~nm} ;-29.7 \pm 1.2 \mathrm{mV})$. Remarkably, GOn aqueous dispersions were stable for up to 6 months of shelf-time, with a global process yield of $74 \%$. This novel method enabled the production of large volumes of highly concentrated $\left(7.5 \mathrm{mg} \mathrm{mL}^{-1}\right)$ GOn aqueous dispersions. Chemical characterization of GOn allowed the identification of characteristic oxygen functional groups, supporting high-power ultrasonication as a fast, efficient, and productive process for reducing GO lateral size, while maintaining the material's chemical features.
\end{abstract}

Keywords: graphene; graphene oxide; particle size; stability; standardization; surface chemistry; nanomaterials

\section{Introduction}

Graphene oxide (GO) with very small lateral dimensions, in the order of $100 \mathrm{~nm}$ or less-commonly designated as nanographene (GOn)—has been attracting increasing attention in the biomedical field, particularly in the area of cancer treatment. Proposed applications include uses as drug carriers, platforms for photothermal and photodynamic therapies, or agents for biological imaging [1-3]. These take advantage of GOn's physicochemical properties in combination with good biocompatibility and low toxicity. GO has been also explored to improve biomaterials' physicochemical and biological properties [4] as well as for its antibacterial effect [5-7].

The size of the GO platelets is a relevant parameter when considering biological systems, since it may affect absorption into the body, penetration into blood vessels, cellular uptake, renal clearance, and selective toxicity $[2,8,9]$. GO is most widely produced using the modified Hummers method, a graphite chemical oxidation/exfoliation process, yielding sheets with lateral dimensions of tens or hundreds of microns. Decreasing the GO lateral dimensions to average values around $100 \mathrm{~nm}$, ideally guaranteeing sufficiently narrow size 
distributions and maintaining the surface chemistry, in terms of degree of oxidation (i.e., avoiding reduction), has been the subject of several studies. These were recently reviewed by Tufano et al. [2] and the main approaches involved can be summarized as:

- Intensive oxidation of graphite by increasing concentration of oxidizing agents or increasing the timescale/cycles of oxidation process

- Centrifugation of GO in aqueous dispersion and separation of the fractions with smaller dimensions

- Breakdown of GO sheets by high-power ultrasonication

- Selective precipitation of larger GO sheets by protonation with organic solvents or by $\mathrm{pH}$ adjustment

- Exfoliation of graphite nanofibers with very small diameter

- Electrical breakdown of graphite by arc-discharge

- Ball milling of graphite in the presence of oxidizing agents

- Electrochemical exfoliation of graphite electrodes

Among these, electrochemical exfoliation has attracted attention for being relatively simple and fast [10]. However, ultrasonic treatment is also of particular interest since it has the potential to be a relatively expedited process that does not imply additional environmentally aggressive reactants or complex manipulation and can be easily scaled up. Ultrasonication is actually already used in combination with the Hummers or Marcano methods of GO production to achieve the final exfoliation and dispersion of GO flakes in liquid medium, albeit most often using low-power bath immersion systems and, therefore, not inducing significant changes in lateral dimensions [11]. The first use of high-power ultrasound treatment for effective reduction of GO's lateral dimensions was recently reported by Méndez-Romero and coworkers [12]. GO was first produced by the modified Hummers method, having been exfoliated for $1 \mathrm{~h}$ in a low-power ultrasonic bath in the last step. A $10 \mathrm{~mL}$ amount of this aqueous GO dispersion (with concentration $3 \mathrm{mg} \mathrm{mL}^{-1}$ ) was then submitted to a high-power ultrasound probe ( $250 \mathrm{~W}$ maximum power) for a maximum time of $4 \mathrm{~h}$ under controlled temperature $\left(18^{\circ} \mathrm{C}\right)$. The product was centrifuged to separate larger particles, but the authors report that the GOn yield from these ultrasonication and centrifugation steps was $90 \%$. Particle sizes below $100 \mathrm{~nm}$ were obtained after $2 \mathrm{~h}$ treatment.

The present work further explores the use of high-power ultrasound treatment for efficiently producing GOn. By using a continuous recirculation system, we show that this method can be used for processing large quantities of aqueous GO dispersion at high concentration. In addition, we produce GOn directly from oxidized graphite (GtO), with no intermediate GO exfoliation step in a low-power ultrasonic bath. The final GOn is compared to GO obtained by the conventional centrifugation approach for lateral dimension reduction.

\section{Materials and Methods}

\subsection{GO Lateral Dimensions Reduction Based on Centrifugation}

Graphene oxide (GO) was prepared by oxidation of graphite powder using the modified Hummers method [13]. In a jacketed glass reactor, $160 \mathrm{~mL}$ of sulfuric acid $\left(\mathrm{H}_{2} \mathrm{SO}_{4}\right)$ and $40 \mathrm{~mL}$ of phosphoric acid $\left(\mathrm{H}_{3} \mathrm{PO}_{4}\right)$ were added to $4 \mathrm{~g}$ of graphite powder (size $\leq 20 \mu \mathrm{m}$, Sigma Aldrich, St. Louis, MO, USA), stirring for $10 \mathrm{~min}$. Then, $24 \mathrm{~g}$ of potassium permanganate $\left(\mathrm{KMnO}_{4}\right)$ was slowly added to the solution, which was heated to $35^{\circ} \mathrm{C}$ and stirred for $2 \mathrm{~h}$. Following this, $600 \mathrm{~mL}$ of $\mathrm{H}_{2} \mathrm{O}$ was gradually added, under stirring. Temperature was controlled using a thermocryostatic bath. To stop the reaction, $26 \mathrm{~mL}$ hydrogen peroxide $\left(\mathrm{H}_{2} \mathrm{O}_{2}\right)$ was added to the mixture. The next day, the solution was decanted and the solid phase was separated from the acidic solution. A centrifugation step was performed at $4000 \mathrm{rpm}$ for $20 \mathrm{~min}$; the solution was redispersed in distilled water, and this protocol was repeated until a neutral supernatant $\mathrm{pH}$ was achieved. To obtain well-exfoliated GO flakes, the pellet collected after the last centrifugation step was redispersed in $300 \mathrm{~mL}$ of distilled water at a concentration of $1 \mathrm{mg} / \mathrm{mL}$ and placed in a conventional ultrasonic bath (Ovan ATM40-3LCD, Barcelona, Spain) with a power density of $30 \mathrm{~W} / \mathrm{L}$ for $4 \mathrm{~h}$. After sonication, 
GO dispersion was centrifuged at 13,000 rpm for $30 \mathrm{~min}$, which enabled the separation of two different phases. The supernatant contained the smallest particles and was recovered for later use [3].

\subsection{GO Lateral Dimensions Reduction Based on High-Power Ultrasonication}

The improved approach relied on a similar oxidation process of graphite powder, as described above, which, after the last washing cycle, was immediately followed by high-power ultrasonication in order to simultaneously exfoliate and breakdown the GO sheets. For this purpose, a high-power ultrasound probe (Hielscher UIP1000hd, $1000 \mathrm{~W}$ maximum power, Teltow, Germany) was used (Figure 1). The aqueous dispersion was made to recirculate continuously with the help of a peristaltic pump (Watson-Marlow 323, Falmouth, UK) through a $40 \mathrm{~mL}$ stainless steel flow cell (Hielscher FC100L1) housing the ultrasound probe, and a glass condenser that worked as a heat exchanger for cooling. The ultrasound power density in the flow cell was $25,000 \mathrm{~W} / \mathrm{L}$, which is coincidentally the same as in the abovementioned work by Méndez-Romero et al., who have also dealt with high-power ultrasonication [12]. Note that in a conventional ultrasound bath, power densities typically range from 10 to $40 \mathrm{~W} / \mathrm{L}$. The cooling fluid was water pumped from a thermocryostatic bath kept at $40^{\circ} \mathrm{C}$. The recirculation flow rate was $400 \mathrm{~mL} / \mathrm{min}$. Samples were collected at different times to evaluate the influence of process duration on GOn size, up to a maximum of $8 \mathrm{~h}$. The final product was collected and was stored without further processing. A total volume of $1000 \mathrm{~mL}$ of GOn dispersion could be processed in this system, with a concentration of $7.5 \mathrm{mg} \mathrm{mL}^{-1}$. It was observed that concentrations above $10 \mathrm{mg} \mathrm{mL}^{-1}$ were difficult to process due to increased viscosity.

Legend:

1 - Stirred tank

2 - Heat exchanger

3 - Flow cell with US probe

4 - Peristaltic pump

5 - Thermochriostatic bath

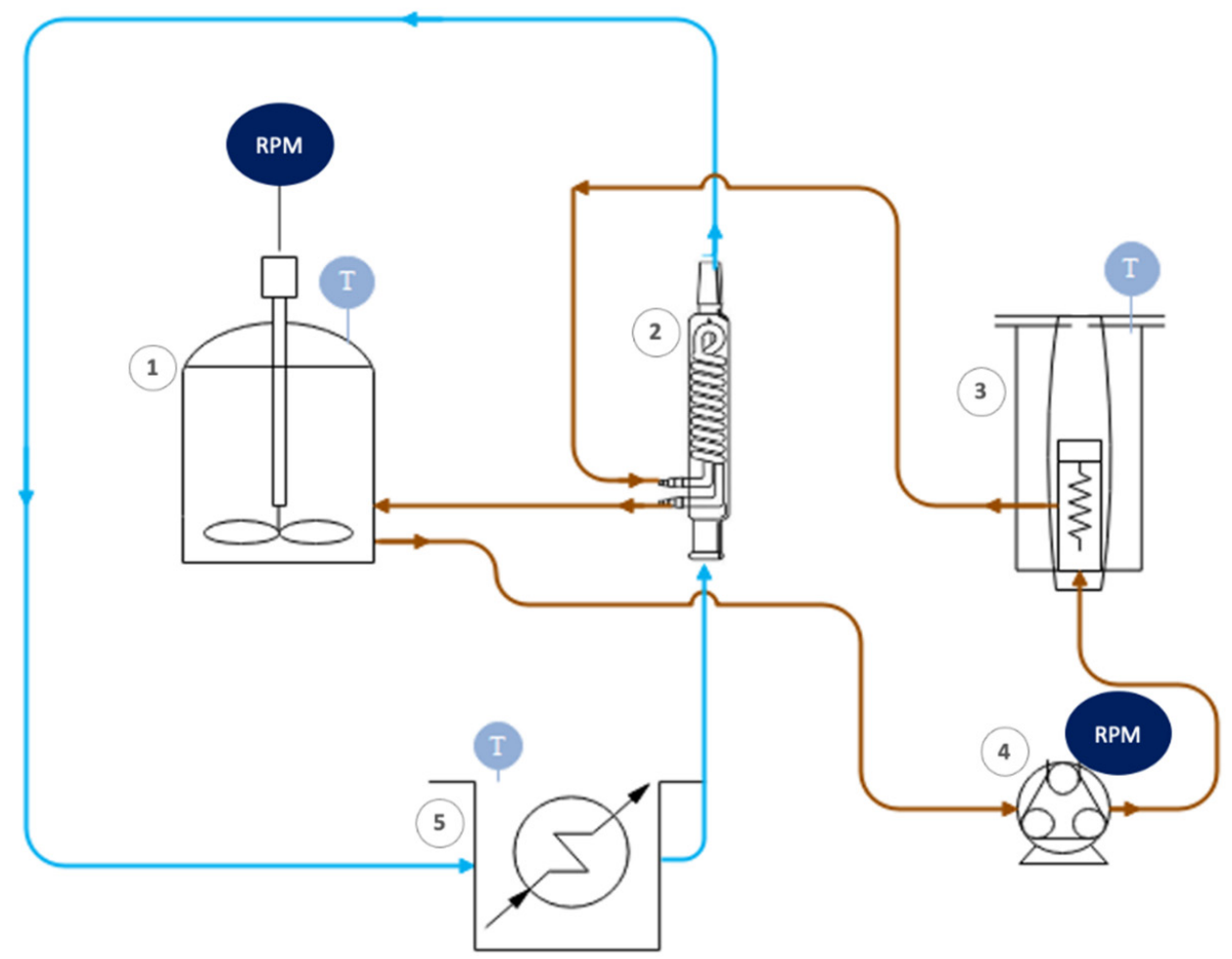

Figure 1. Schematic representation of the recirculation system for GOn production by high-power ultrasonication.

Figure 2 depicts schematically the steps involved in the two production methods used here, which differ in the way how GtO is processed to obtain small size GO. In the ensuing text, we shall designate as "GOn" the graphene oxide produced by high-power ultrasound, and as GO the graphene oxide obtained by the conventional low-power ultrasound plus centrifugation method. 


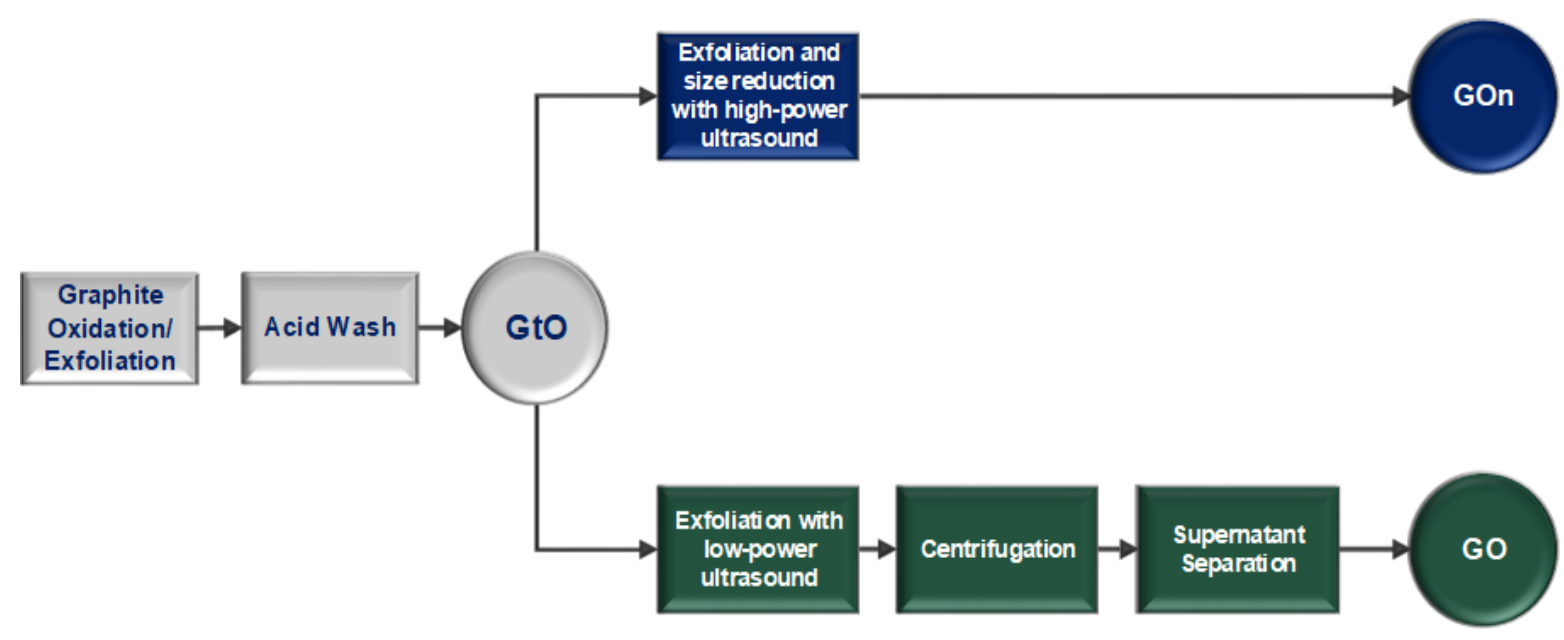

Figure 2. Flow diagram for the new high-power ultrasound method and the conventional centrifugation method for production of GO with small lateral dimensions.

\subsection{Transmission Electron Microscopy (TEM)}

GO and GOn aqueous dispersions were analyzed by transmission electron microscopy (TEM, JEOL JEM 1400 TEM, Tokyo, Japan) to evaluate their morphology and determine lateral dimensions. For this, $10 \mu \mathrm{L}$ of each sample (concentration of $50 \mu \mathrm{g} \mathrm{mL}^{-1}$ ) were deposited on Formvar/carbon film-coated 300 mesh nickel grids (Electron Microscopy Sciences, Hatfield, PA, USA) and left standing for $1 \mathrm{~min}$, followed by excess liquid removal with filter paper. Nanomaterial sizes were determined using ImageJ software (version 1.53) by measuring lateral dimensions of platelets using several acquired TEM images [14].

\subsection{Zeta Potential Measurements}

Zeta potentials of GO and GOn aqueous dispersions at a concentration of $25 \mu \mathrm{g} \mathrm{mL} \mathrm{L}^{-1}$ were measured in a Zetasizer Nano-ZS (Malvern Instruments, Worcestershire, UK) using a disposable Zetasizer cuvette (Malvern Instruments, Worcestershire, UK). All samples were analyzed in triplicate at room temperature. Results are reported as mean and standard deviation.

\subsection{Fourier Transform Infrared (FTIR) Spectroscopy}

GO and GOn dehydrated samples were examined using a VERTEX 70 FTIR spectrometer (Bruker, Karlsruhe, Germany) in transmittance mode. Samples were analyzed in ATR mode using a A225/Q PLATINUM ATR Diamond crystal with single reflection accessory at room temperature (Bruker, Karlsruhe, Germany). Infrared spectra were recorded over the wavenumber range between 4000 and $400 \mathrm{~cm}^{-1}$ and 64 scans were averaged at resolution of $4 \mathrm{~cm}^{-1}$.

\subsection{X-ray Photoelectron Spectroscopy (XPS)}

X-ray photoelectron spectroscopy (XPS, Kratos Axis Ultra HSA, Manchester, UK) analysis was performed at CEMUP (Centro de Materiais da Universidade do Porto, Porto, Portugal). Data acquisition was performed using a monochromator Al X-ray source operating at $15 \mathrm{kV}(90 \mathrm{~W})$. The survey XPS spectra were acquired, with pass energy (PE) of $80 \mathrm{eV}, 1 \mathrm{eV}$ step size, $200 \mathrm{~ms}$ dwell time and averaged from two scans. High-resolution C1s and O1s XPS spectra were acquired averaging five scans, with PE of $40 \mathrm{eV}, 0.1 \mathrm{eV}$ step size, $1500 \mathrm{~ms}$ (for C1s), and $1000 \mathrm{~ms}$ (for O1s) dwell time. Spectra were then analyzed using CasaXPS software (Casa Software Ltd., Teignmouth, UK). The contribution of the electric charge was corrected by calibrating all samples to the carbon peak reference at a binding energy of $284.6 \mathrm{eV}$. 


\subsection{Thermogravimetric Analysis (TGA)}

Weight loss of GO and GOn (sample amounts of 4-4.5 mg) was determined by thermogravimetric analysis (TGA) (Netzsh STA 449 F3 Jupiter, Selb, Germany) under a constant temperature increase. All thermograms were obtained under nitrogen flow and collected between 30 and $1000{ }^{\circ} \mathrm{C}$ at a heating rate of $10{ }^{\circ} \mathrm{C} \mathrm{min}^{-1}$. Results are presented as percentage $(\%)$ of weight loss.

\subsection{X-ray Diffraction Analysis (XRD)}

GBM films were analyzed by XRD using a Rigaku SmartLab diffractometer (Tokyo, Japan). For data acquisition, an operating voltage of $45 \mathrm{kV}$ and $200 \mathrm{~mA}$ under $\mathrm{Cu} \mathrm{K} \alpha$ radiation of wavelength $\lambda \sim 1.540 \AA$, in a Bragg-Brentano geometry, was used. GO and GOn films were analyzed in the range of $5-50$ theta $(2 \theta)$ with a step of $0.01^{\circ}$ and measured in a rotative system $\left(30 \mathrm{deg} \mathrm{min}^{-1}\right)$ to increase the crystallite size statistics. Crystallites interlayer d-spacing was extracted from the Rigaku PDXL XRD analysis software based on the Bragg's law equation.

\subsection{Raman Spectroscopy}

Raman spectroscopy was performed to characterize vibrational GOn modes. Spectra were acquired using a Raman confocal microscope (WITec alpha300 R, Ulm, Germany). Excitation was provided by a $532 \mathrm{~nm}$ laser. Measurements were performed using film samples. Each spectrum is an average of 30 scans and was corrected at the baseline and smoothed.

\section{Results and Discussion}

\subsection{Morphological Features and Dispersion Stability}

Figure 3 shows TEM images and particle size distributions for GO after low-power ultrasound exfoliation of $\mathrm{GtO}, \mathrm{GO}$ collected from the supernatant after centrifugation of the previous dispersion, and GOn obtained by direct high-power ultrasonication of GtO.
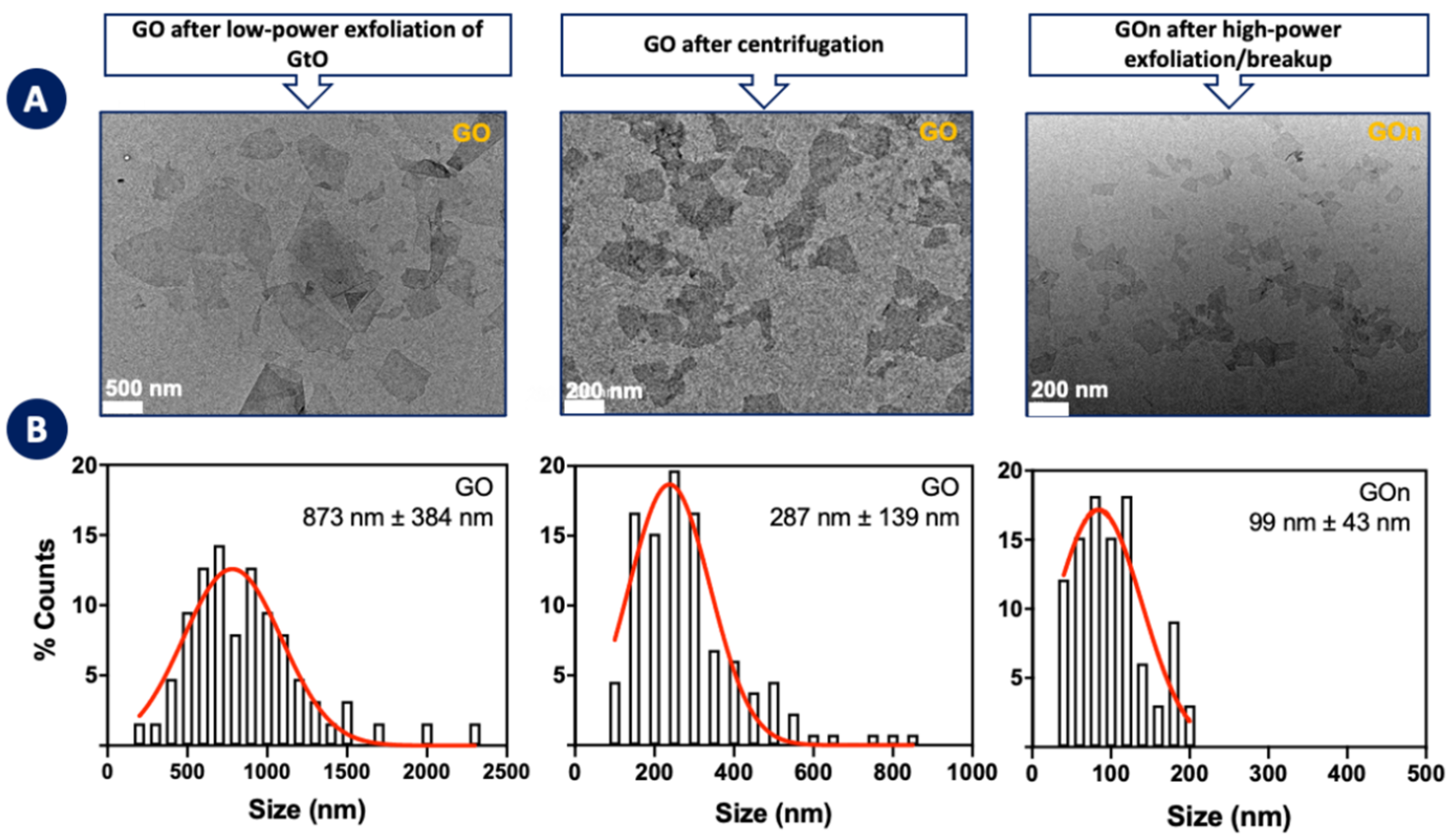

Figure 3. Morphology of GO and GOn. (A) Representative TEM images of GO and GOn aqueous dispersions and (B) respective particle size distributions and average sizes and standard deviations, as determined from TEM images. 
Centrifugation allows separating GO sheets with $287 \mathrm{~nm}$ of average lateral dimensions, lower than the $873 \mathrm{~nm}$ of the original GO dispersion, but the global yield, from GtO to the final GO, is only $17 \%$. On the other hand, high-power ultrasonication of GtO allows obtaining GO sheets with lower lateral sizes (average length $99 \mathrm{~nm}$ ), and a much higher global yield of $74 \%$, since all the treated material is usable, except for process losses (material not properly removed from tubing connections, for instance).

Figure 4 shows the evolution of particle size along the high-power ultrasonication step. As noted before, the starting material (time $0 \mathrm{~h}$ ) is the unexfoliated oxidized graphite. Figure $4 \mathrm{~A}$ presents TEM images of the starting particles and of the product obtained after $8 \mathrm{~h}$ ultrasonication, illustrating the magnitude of lateral size reduction. This process is able to break down particle sizes rapidly and uniformly, eliminating the need for separation of the smaller sizes from the rest of the material. It is noticeable that most of the lateral dimension reduction occurs in the first $2 \mathrm{~h}$ (Figure 4B). This fast initial effect was also reported in the abovementioned work by Méndez-Romero and coworkers [12]. The initially large concentration of defects in graphene oxide's basal plane (lower dissociation energy related to $\mathrm{sp}^{3}$ bonding) facilitates sheet breakup caused by the high shear forces associated with ultrasound's acoustic cavitation.
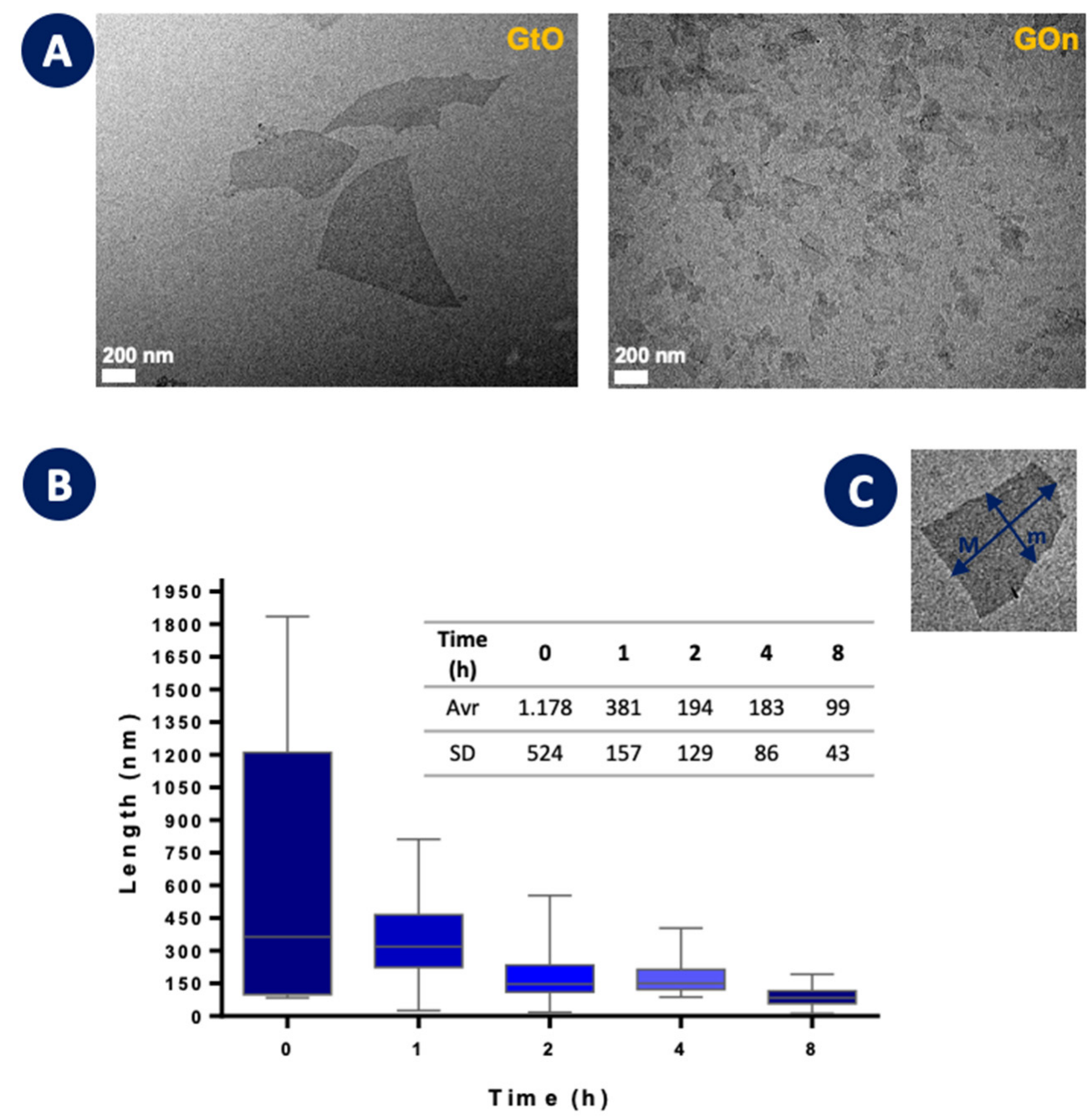

Figure 4. Effect of high-power ultrasonication time on the particle size of GOn. (A) TEM images comparing starting GtO particles and the GOn sheets obtained after $8 \mathrm{~h}$ of high-power ultrasonication. (B) Box plot of particle sizes and average and standard deviations (SD). (C) Representation of the major $(\mathrm{M})$ and minor $(\mathrm{m})$ axis used for calculation of average lateral dimensions from TEM images. 
Decreasing GO's lateral dimensions is expected to lead to higher charge density, due to higher edge-to-area ratio (cationic carboxylic groups are more abundant along the sheet edges). As a consequence, zeta potential as well as colloidal stability tends to be higher [15]. Zeta potential measurements demonstrated that both GO and GOn were negatively charged colloidal particles. As seen in Table 1, we found that high-power ultrasonicated GOn displayed a greater negative surface charge $(-39.9 \pm 2.2 \mathrm{mV})$ as compared to centrifuged GO $(-29.7 \pm 1.2 \mathrm{mV})$. This higher density of negative electrostatic charges resulted in increased colloidal stability of GOn aqueous dispersions (Figure 5), which exhibited long term stability, even after 6 months storage. On the other hand, GO dispersions were already extensively separated after 6 months (Figure 5), having started to visibly sediment after only one week. Even more striking is the fact that the dispersion concentration was significantly higher for GOn than for GO $\left(7.5\right.$ and $1 \mathrm{mg} \mathrm{mL}^{-1}$, respectively). Méndez-Romero and coworkers reported that zeta potential did not tend to change with lateral size for their GOn produced by high-power ultrasonication, though they did not evaluate colloidal stability [12].

Table 1. Zeta potential measurements of GO and GOn aqueous dispersions.

\begin{tabular}{cc}
\hline GBM & Surface Charge $(\mathbf{m V})$ \\
\hline GO & $-29.7 \pm 1.2$ \\
GOn & $-39.9 \pm 2.2$ \\
\hline
\end{tabular}
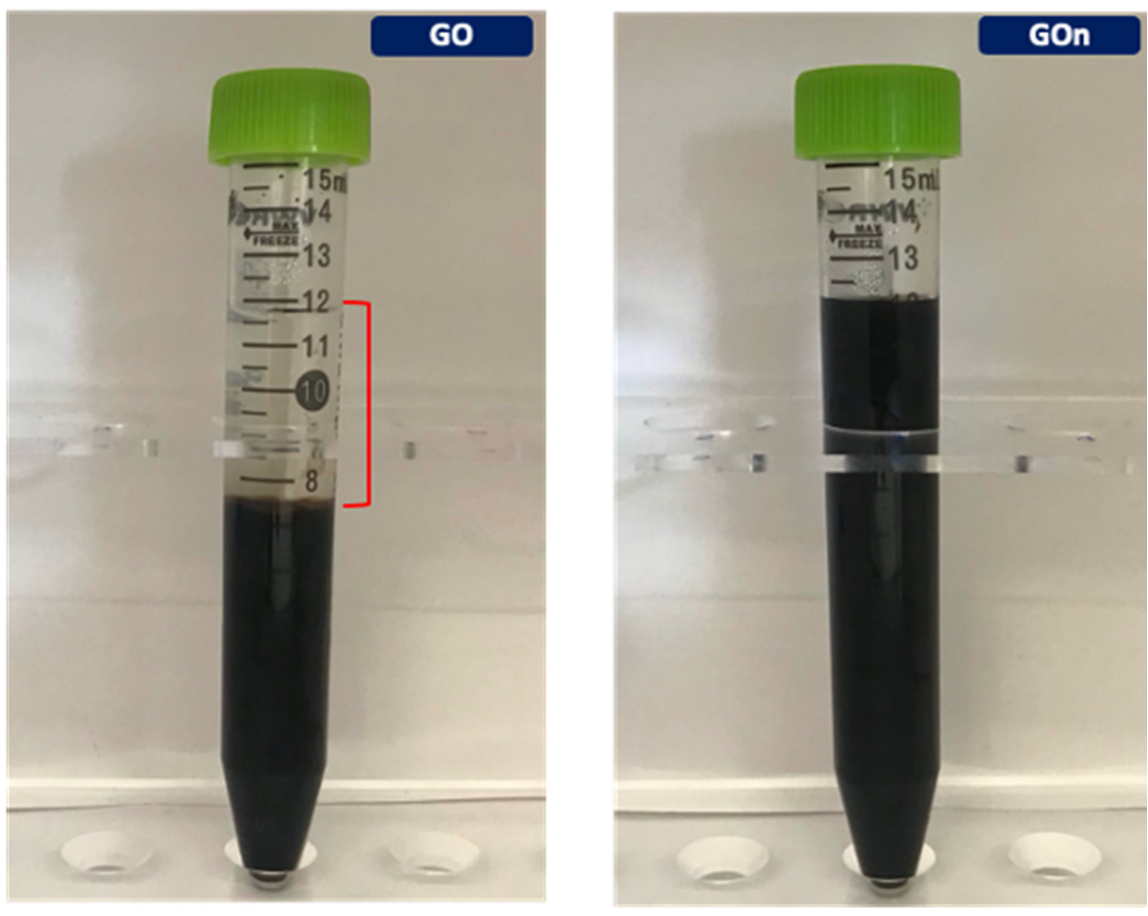

Figure 5. GO (left) and GOn (right) dispersions after 6 months storage at rest. The red bracket on the left image shows the height of clear supernatant liquid formed in the GO dispersion.

Generally, smaller average particle size and coefficient of variation (ratio of the standard deviation to the mean particle size) together with lower zeta potential values have been identified as critical parameters to demonstrate dispersion stability of GO materials, thus favoring the production of homogeneous GO dispersions at larger scales and with improved product quality $[16,17]$. Overall, high-power ultrasonication enabled the production of GO nanosheets with smaller average particle size ( 99 versus $287 \mathrm{~nm}$ for centrifuged GO) and slightly lower smaller coefficient of variation ( $43 \%$ versus $48 \%$ ). Additionally, GO 
dispersions exhibiting a zeta potential value lower than $-30 \mathrm{mV}$ have been described as highly stable $[18,19]$, further supporting dispersion stability improvement reported herein.

\subsection{Chemical Properties}

Fourier transform infrared (FTIR) spectroscopy was used to analyze the contribution of oxygen functionalities on the surface of GO and GOn (Figure 6). Herein, a broad band could be identified for both GO types in the wavenumber range of 3000 and $3600 \mathrm{~cm}^{-1}$, corresponding to $\mathrm{O}-\mathrm{H}$ stretching vibrations, which are characteristic of adsorbed water molecules and hydroxyl and carboxyl groups $[3,14]$. A peak was evident at around $1725 \mathrm{~cm}^{-1}$ in both GO and GOn spectra, which is assigned to $\mathrm{C}=\mathrm{O}$ stretching vibrations, demonstrating the presence of carbonyl and carboxyl groups $[3,14,20]$. The stretching of cyclic alkene $(C=C)$ was observed at approximately $1610 \mathrm{~cm}^{-1}$, which results from the unoxidized graphitic backbone [20-22]. C-O stretching vibrations attributed to ethers were present at around 1140 and $1020 \mathrm{~cm}^{-1}$, and $\mathrm{C}-\mathrm{O}$ bending vibrations of epoxides were found at around $858 \mathrm{~cm}^{-1}[3,14]$. Further characterization regarding carbon and oxygen contents and chemical functional groups quantification were performed by other techniques, which is presented next.

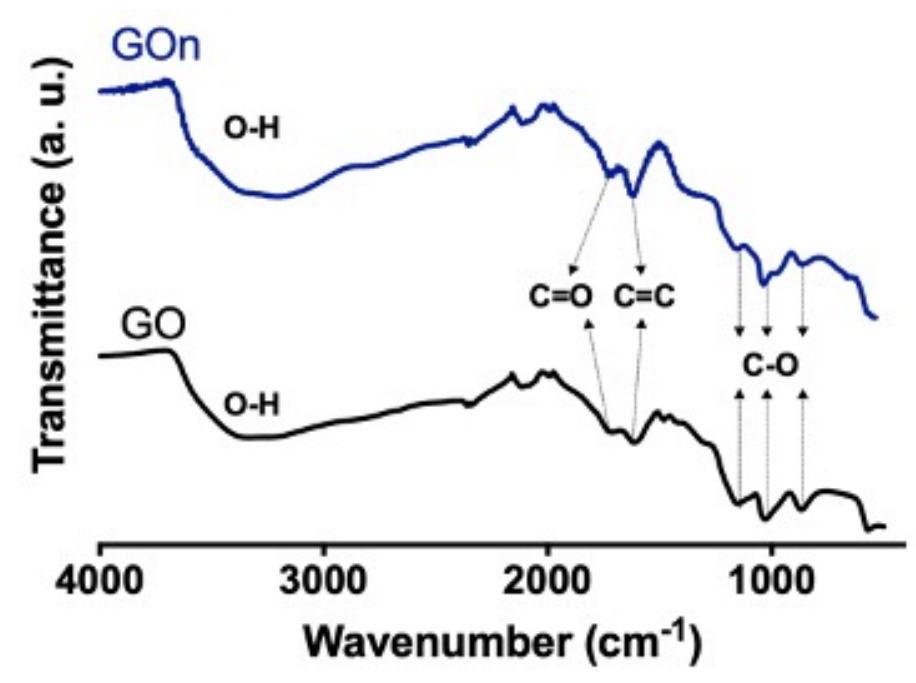

Figure 6. FTIR spectra of GO (black line) and GOn (blue line), showing the contribution of surface functionalities.

GO and GOn functionalization degree and thermal stability were assessed by TGA. Results are shown in Figure 7, displaying the weight loss during heating. Thermograms revealed two main weight loss steps for both materials. The first weight loss occurred between $142-240{ }^{\circ} \mathrm{C}$, which is attributed to the loss of reactive oxygen-containing functional groups, namely carboxyl and epoxy $[14,23]$. The second weight loss step occurred between $225-600{ }^{\circ} \mathrm{C}$, corresponding to the combustion of carbon skeleton and pyrolysis of more stable functionalities like carbonyls and residual hydroxyls [24]. At $240{ }^{\circ} \mathrm{C}, \mathrm{GO}$ presented a wt. $\%$ loss of $35.1 \%$, while GOn presented a wt. $\%$ loss of $43.6 \%$. At $600{ }^{\circ} \mathrm{C}, \mathrm{GO}$ presented a wt. $\%$ loss of $56.8 \%$, while GOn presented a wt. $\%$ loss of $60.0 \%$. Such results demonstrate that GOn might be slightly more reduced than GO. Our method involves recirculation of $\mathrm{GtO}$ dispersions through a high-power sonication probe for $8 \mathrm{~h}$. Even though the fluid is cooled during the entire process, as the material contacts with the tip several times, slight chemical reduction might be occurring. In addition, in GO production, there is a highspeed sonication step $(13,000 \mathrm{rpm})$ that allows collecting the material in the supernatant composed of the more water-stable particles, therefore with more oxygen-containing functional groups. 


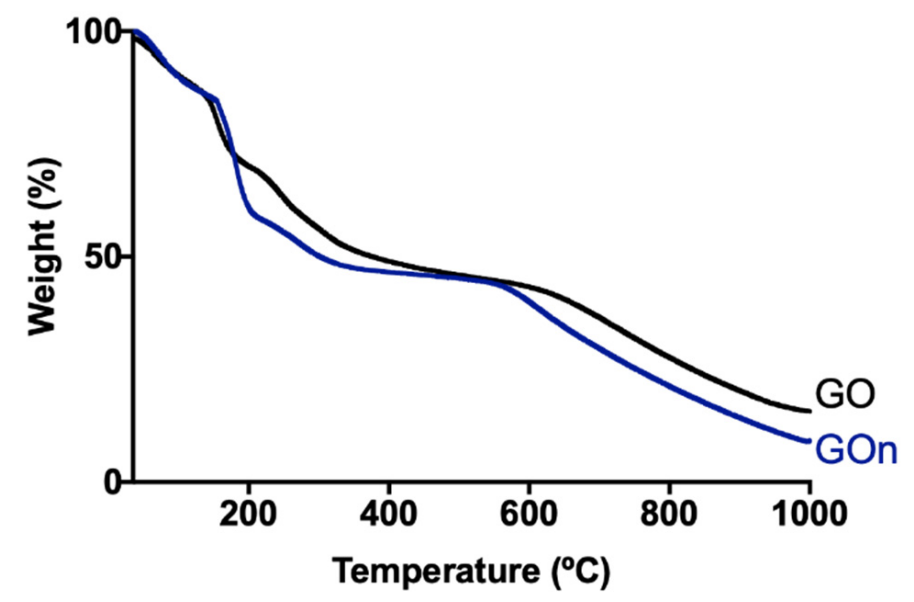

Figure 7. Thermal decomposition of GOn and GO. TGA curves and weight loss values for GOn (blue line) and GO (black line).

X-ray photoelectron spectroscopy (XPS) analyses allowed the quantification of the oxidation degree and surface functionalization of GO and GOn (Figure 8, Table 2). GO exhibited a $\mathrm{C}$ at. \% of 62.1 and a $\mathrm{O}$ at. \% of 32.0, whereas GOn presented a slightly higher $\mathrm{C}$ at. $\%$ of 66.3 , together with a $\mathrm{O}$ at. $\%$ of 30.7 (Table 2). These results indicate successful oxidation and introduction of oxygen functionalities at the surface of GO and GOn. Moreover, similar C/O ratios were determined for both GO types (1.94 and 2.16 for GO and GOn, respectively), suggesting a slightly higher oxidation degree for GO than GOn (Table 2). As previously mentioned, the higher energy applied during contact with the high-power sonication probe in multiple recirculation cycles could have contributed to a slight reduction in GOn. Similarly, Méndez-Romero and coworkers reported a slight increase in C/O ratio, ranging from 1.95 to 2.01, for their GOn exposed to increasing times of high-power ultrasonication [12]. It is important to notice that, in our method, we produce GOn directly from GtO, contrary to the previously mentioned work, where previously exfoliated GO (not GtO) is size reduced.
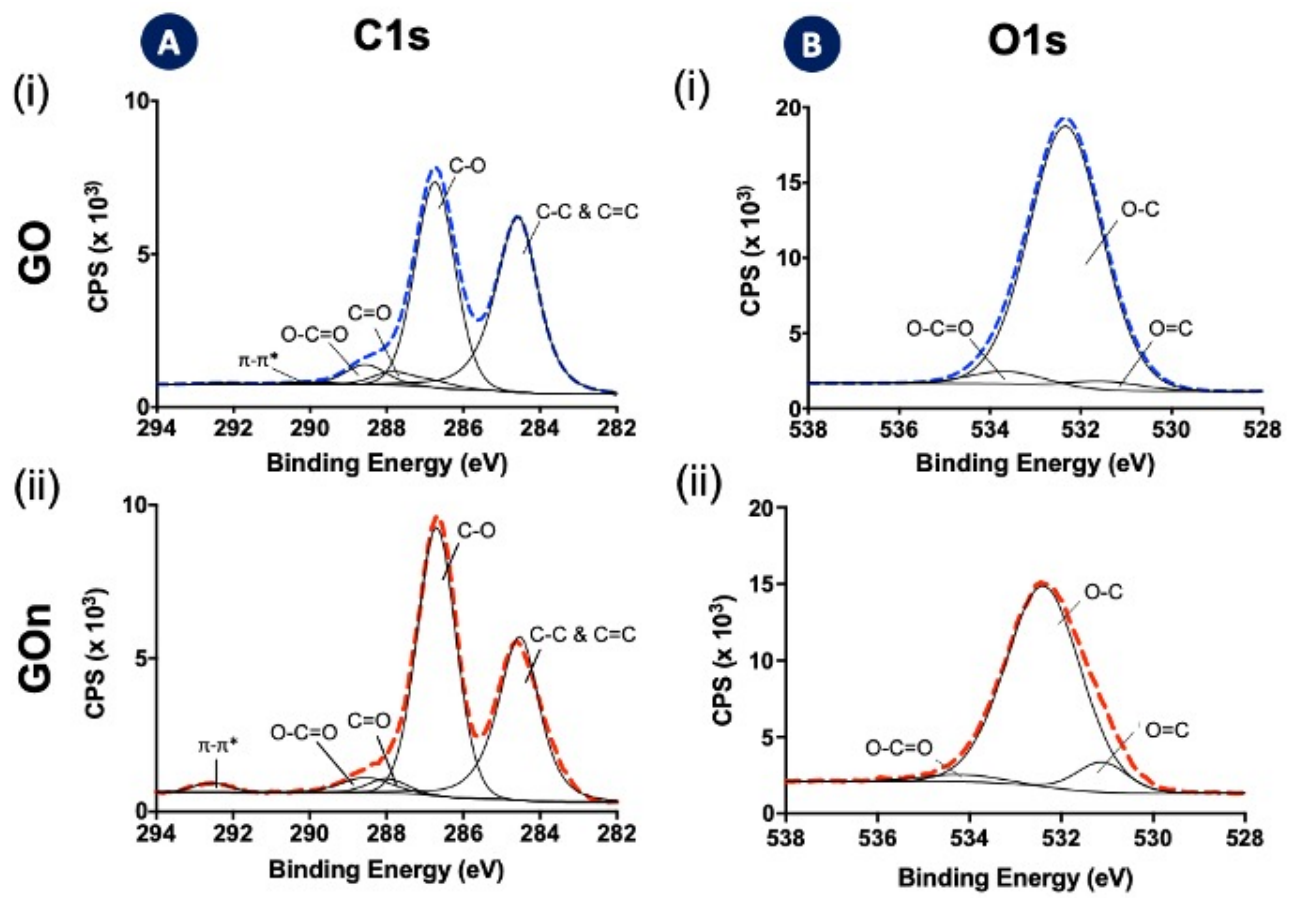

Figure 8. XPS analysis of (i) GO and (ii) GOn. Deconvolution of high-resolution (A) C1s and (B) O1s spectra enabled the quantification of functional groups on graphene backbone. 
Table 2. Atomic composition of GO and GOn and content of $\mathrm{C} 1$ s and $\mathrm{O} 1$ s chemical functional groups resulting from XPS spectra fitting.

\begin{tabular}{ccccc}
\hline Elemental at. \%/Chemical Group & Binding Energy (eV) & $\begin{array}{c}\text { GO } \\
\mathbf{( \% )}\end{array}$ & $\begin{array}{c}\text { GOn } \\
\mathbf{( \% )}\end{array}$ \\
\hline & $\mathrm{C} / \mathrm{O}$ ratio & - & 1.94 & 2.16 \\
\hline $\mathrm{C} 1 \mathrm{~s}$ & - & 62.1 & 66.3 \\
$\mathrm{O} 1 \mathrm{~s}$ & - & 32.0 & 30.7 \\
\hline & $\mathrm{C}-\mathrm{C}$ and C=C & 284.5 & 45.5 & 38.4 \\
$\mathrm{C} 1 \mathrm{~s}$ & $\mathrm{C}-\mathrm{O}$ & 286.7 & 44.9 & 53.2 \\
$($ at. $\%)$ & $\mathrm{C}=\mathrm{O}$ & 287.9 & 4.2 & 2.8 \\
& $\mathrm{O}=\mathrm{C}-\mathrm{O}$ & 288.5 & 4.5 & 3.9 \\
& $\pi-\pi^{*}$ & 292.6 & 0.93 & 1.8 \\
\hline $\mathrm{O} 1 \mathrm{~s}$ & $\mathrm{C}=\mathrm{O}$ & 531.2 & 3.1 & 5.7 \\
$($ at. $\%)$ & $\mathrm{C}-\mathrm{O}$ & 532.5 & 92.4 & 92.5 \\
& $\mathrm{O}=\mathrm{C}-\mathrm{O}$ & 534 & 4.5 & 1.9 \\
\hline
\end{tabular}

Analysis of C1s spectra of both GO and GOn showed two large peaks, which were then deconvoluted in five peaks centered at 284.5, 286.7, 287.9, 288.5, and 292.6 eV (Figure 8A, Table 2), which can be attributed to formation of the following:

1. $\mathrm{sp}^{2}$ and $\mathrm{sp}^{3}$ hybridizations of carbon $(\mathrm{C}-\mathrm{C}$ and $\mathrm{C}=\mathrm{C}, \mathrm{C} 1 \mathrm{~s}$ at. $\%=45.5$ for $\mathrm{GO}$ and $\mathrm{C} 1 \mathrm{~s}$ at. $\%=38.4$ for GOn) in the graphitic backbone;

2. single bonds between carbon and oxygen $(\mathrm{C}-\mathrm{O})$ in hydroxyls and ethers $(\mathrm{C} 1 \mathrm{~s}$ at. $\%=$ 44.9 for GO and C1s at. \% = 53.2 for GOn);

3. double bonds between carbon and oxygen $(\mathrm{C}=\mathrm{O})$, indicating the presence of carbonyl groups ( $\mathrm{C} 1 \mathrm{~s}$ at. $\%=4.2 \%$ for $\mathrm{GO}$ and $\mathrm{C} 1 \mathrm{~s}$ at. $\%=2.8 \%$ for $\mathrm{GOn}$ );

4. multiple bonds between carbon and oxygen $(\mathrm{O}=\mathrm{C}-\mathrm{O})$, indicating the occurrence of carboxyls (C1s at. $\%=4.5$ for $\mathrm{GO}$ and $\mathrm{C} 1 \mathrm{~s}$ at. $\%=3.9$ for GOn); and

5. $\pi-\pi^{*}$ bonds due to the presence of delocalized $\pi$ electrons in the graphene lattice ( $\mathrm{C} 1 \mathrm{~s}$ at. $\%=0.93$ for GO and C1s at. $\%=1.8$ for GOn) $[3,14,25]$.

The high-resolution O1s spectra were deconvoluted in three peaks centered at 531.2, 532.5, and $534 \mathrm{eV}$ (Figure 8B, Table 2), which can be also attributed to formation of the following:

1. $\mathrm{O}=\mathrm{C}$ bonds present in carbonyl and carboxyl groups (O1s at. $\%=3.1$ for $\mathrm{GO}$ and $\mathrm{O} 1 \mathrm{~s}$ at. $\%=5.7$ for GOn);

2. $\mathrm{O}-\mathrm{C}$ bonds in hydroxyl groups and ethers $(\mathrm{O} 1 \mathrm{~s}$ at. $\%=92.4$ for $\mathrm{GO}$ and $\mathrm{O} 1 \mathrm{~s}$ at. $\%=$ 92.5 for GOn); and

3. $\mathrm{O}-\mathrm{C}$ bonds from carboxyls $(\mathrm{O}=\mathrm{C}-\mathrm{O}, \mathrm{O} 1 \mathrm{~s}$ at. $\%=1.9$ for $\mathrm{GO}$ and $\mathrm{O} 1 \mathrm{~s}$ at. $\%=4.5$ for GOn) $[3,14,26]$.

The relative percentage of chemical bonds found in both $\mathrm{C} 1 \mathrm{~s}$ and O1s spectra are in accordance. The analysis of the deconvoluted spectra showed that GO (Figure 8A) and GOn (Figure 8B) were well oxidized, as demonstrated by the existence of carbon atoms in functional groups (hydroxyl, carbonyl, and carboxyl) with $\mathrm{C}-\mathrm{O}$ bonds dominating the surface chemistry. Despite a slightly lower abundance of $\mathrm{C}-\mathrm{O}$ bonds being found in the $\mathrm{C} 1 \mathrm{~s}$ spectra of GOn (Figure 8A), which resulted in a higher abundance of $\mathrm{C}-\mathrm{C}$ bonds compared to GO, the analysis of the O1s spectra (Figure $8 \mathrm{~B}$ ) showed similar amounts of oxygen atoms involved in $\mathrm{C}-\mathrm{O}$ bonds between both $\mathrm{GO}$ materials. Altogether, these results demonstrate that both GO and GOn exhibited high degree of oxidation, in accordance to other studies about the quality of GO materials reporting C/O ratios in the range between 1.94 and $2.6[12,14,23]$.

The materials have also been characterized by XRD, showing typical spectra with GO presenting a $2 \theta$ angle of $10.26^{\circ}$ (Figure $9 \mathrm{~A}$ ) and GOn presenting a value of $10.05^{\circ}$ (Figure 9B). Interlayer spacing for GO was of $8.8 \AA$, while GOn presented an interlayer 
spacing of $8.6 \AA$. This confirms full graphite oxidation and successful exfoliation into GO or GOn [27].
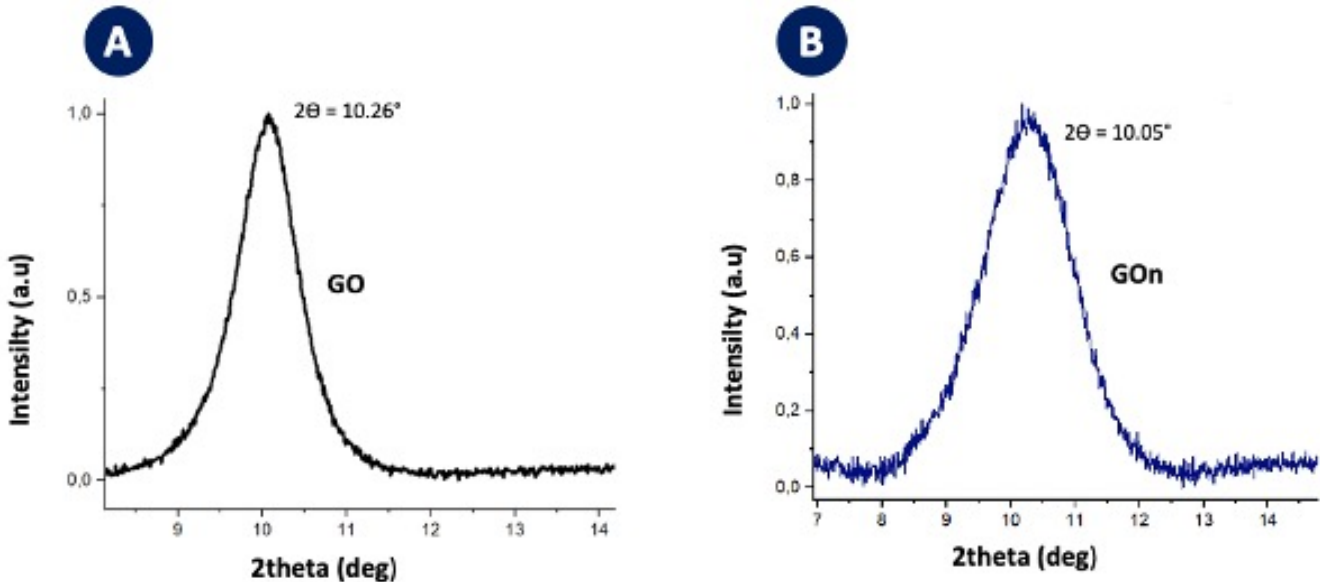

Figure 9. XRD patterns of (A) GO and (B) GOn, corresponding to characteristic network reflections.

Raman spectroscopy was used to further characterize the produced GO types. Graphenebased materials typically present spectra with marked D and G bands [28]. The D peak results from the presence of vacancies or dislocations in the graphene basal plane and at its edge, being therefore related to the presence of structural defects [28,29]. Raman spectra obtained demonstrated the appearance of defects in the crystal structure of graphene, as seen by the D band (Figure 10), which was observed at $1355 \mathrm{~cm}^{-1}$ for GO after low-power exfoliation of GtO, $1358 \mathrm{~cm}^{-1}$ for GO after centrifugation, and $1365 \mathrm{~cm}^{-1}$ for GO after high-power exfoliation of $\mathrm{GtO}$ (Table 3 ). The disorder $\mathrm{D}$ band is a typical feature of graphene oxide as a result of oxidation $[14,23,28]$. The $\mathrm{G}$ band, which is related to the in-plane vibration of $\mathrm{sp}^{2}$ hybridized carbon atoms [28,29], was also present in all samples (Figure 10), being identified at $1605 \mathrm{~cm}^{-1}$ for GO after low-power exfoliation of $\mathrm{GtO}, 1600 \mathrm{~cm}^{-1}$ for GO after centrifugation, and $1602 \mathrm{~cm}^{-1}$ for GO after high-power exfoliation of GtO (Table 3).

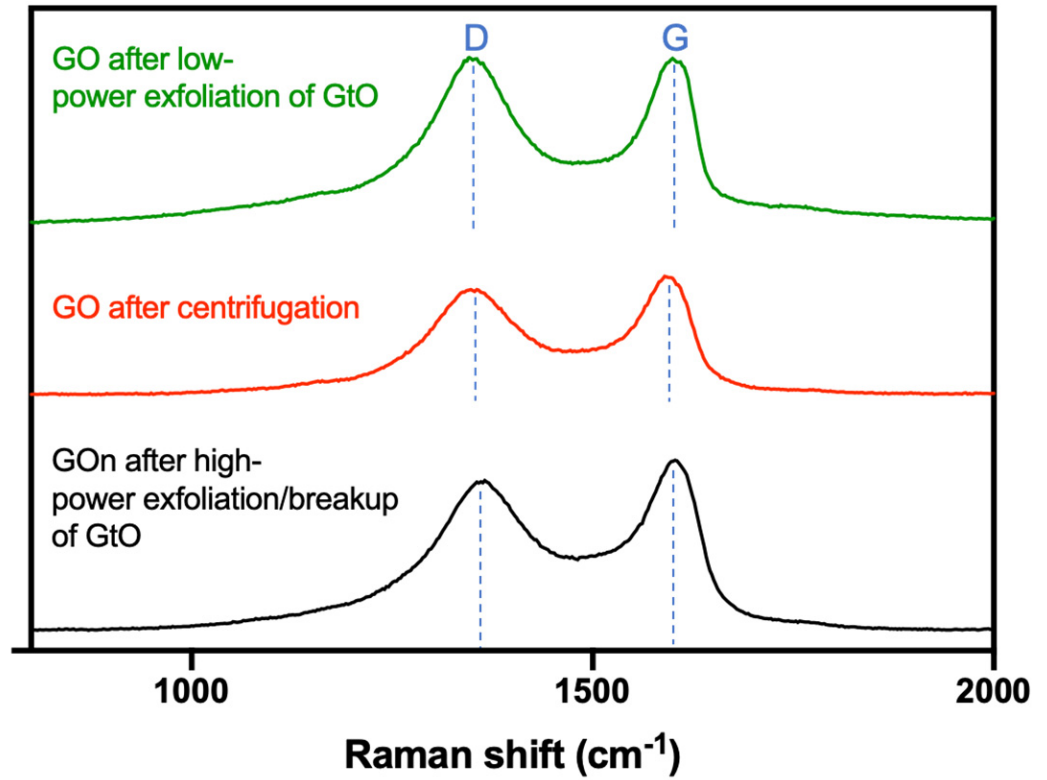

Figure 10. Raman spectra of GO after low-power exfoliation, GO after centrifugation, and GOn after high-power exfoliation/breakup of GtO. 
Table 3. Position of the band $\left(\mathrm{cm}^{-1}\right)$ from Raman to GO after low-power exfoliation, GO after centrifugation and GOn after high-power exfoliation/breakup pf $\mathrm{GtO}$ and respective $\mathrm{I}_{\mathrm{D}} / \mathrm{I}_{\mathrm{G}}$ band ratios.

\begin{tabular}{cccc}
\hline Samples & D Band & G Band & $\boldsymbol{I}_{\mathbf{D}} / \mathbf{I}_{\mathrm{G}}$ Band \\
\hline GO after low-power exfoliation of GtO & 1355 & 1605 & 0.998 \\
GO after centrifugation & 1358 & 1600 & 0.968 \\
GO after high-power exfoliation/breakup of GtO & 1365 & 1602 & 0.880 \\
\hline
\end{tabular}

Ratios between the bands can provide further information on materials structure. The $I_{\mathrm{D}} / I_{\mathrm{G}}$ ratio is related to the amount of defects present in the graphitic structure [29]. The $I_{\mathrm{D}} / I_{\mathrm{G}}$ ratio was highest for $\mathrm{GO}$ after low-power exfoliation of $\mathrm{GtO}$, but decreased as a result of high-power ultrasonication (0.998 versus 0.880 , Table 3 ). High-power sonication has been reported to increase the number of grain boundaries, as particle size is reduced, therefore an increase in $I_{\mathrm{D}} / I_{\mathrm{G}}$ ratio would be expected. However, we have observed that for our particular method, the C/O for GOn is slightly smaller than for GO. This might have resulted in the presence of less defects in GOn basal plane carbon structure, derived from oxygen-containing functional groups presence, contributing to $I_{\mathrm{D}} / I_{\mathrm{G}}$ ratio reduction [12].

\section{Conclusions}

In this work, we reported the use of high-power ultrasonication for one-step graphite oxide exfoliation and breakup in order to produce graphene oxide sheets with very small lateral dimensions. We characterized the impact of this process on the physicochemical properties of the product, comparing it to the material obtained via centrifugation of conventionally produced GO (modified Hummers method followed by low-power ultrasonic exfoliation). The continuous recirculation setup used allowed for processing large amounts of highly concentrated $\mathrm{GtO}$ dispersion (up to $1 \mathrm{~L}$ at $7.5 \mathrm{mg} / \mathrm{mL}$ ) at a controlled temperature, producing GOn with average lateral dimensions of $99 \mathrm{~nm}$ with a process yield of $74 \%$. This particle size reduction method resulted in more stable GO nanocolloids, withstanding a 6-month period of shelf-life owing to the higher charge density, which was demonstrated by a more negative zeta potential of GOn as opposed to the GO sheets separated by centrifugation. Oxygen-containing functional groups were identified in similar quantities for both GO types, indicating that the size breakup treatment did not affect the degree of oxidation. This shows that high-power ultrasound treatment is an effective, productive, and expedited method for manufacture of nanocolloidal GO that can easily be scaled up for industrial production.

Author Contributions: Conceptualization, L.T., R.C.-A., F.D.M. and A.M.P.; Methodology, L.T., R.C.A., D.B., J.S., A.P., F.D.M., A.M.P.; Validation, L.T., F.D.M. and A.M.P.; Formal analysis, L.T., R.C.-A., D.B., J.S., F.D.M. and A.M.P.; Investigation, L.T., R.C.-A., F.A.L.S.S., J.S. and D.B.; Resources, A.P., F.D.M. and A.M.P.; Data curation, L.T., R.C.-A., D.B., J.S., F.D.M. and A.M.P.; Writing-original draft preparation, L.T., R.C-A., J.S., F.D.M. and A.M.P.; Writing-review and editing, R.C.-A., J.S., A.P., F.D.M. and A.M.P.; Supervision, A.P., F.D.M. and A.M.P.; Project administration, F.D.M. and A.M.P.; Funding acquisition, F.D.M. and A.M.P. All authors have read and agreed to the published version of the manuscript.

Funding: This work was financed by FEDER funds through the COMPETE 2020-Operacional Programme for Competitiveness and Internationalisation (POCI), Portugal 2020, and by national funds (PIDDAC) through FCT/MCTES in the framework of the project POCI-01-0145-FEDER-031143, and Base Funding-UIDB/00511/2020 of the Laboratory for Process Engineering, Environment, Biotechnology and Energy_LEPABE. Authors would also like to thank the support of i3S Scientific Platforms and respective funding: HEMS, member of the national infrastructure PPBI—Portuguese Platform of Bioimaging: POCI-01-0145-FEDER-022122; and Biointerfaces and Nanotechnology (BN) Laboratory, Portuguese Funds through FCT, UID/BIM/04293/2019. Artur Pinto thanks the Portuguese Foundation for Science and Technology (FCT) for the financial support of his work contract through the Scientific Employment Stimulus_Individual Call—[CEECIND/03908/2017]. 
Institutional Review Board Statement: Not applicable.

Informed Consent Statement: Not applicable.

Data Availability Statement: The data presented in this study are available on request from the corresponding author.

Acknowledgments: Authors would like to acknowledge Rui Fernandes and Ana Rita Malheiro from the Histology and Electron Microscopy Service (HEMS) of i3S, Porto, for the assistance with TEM studies; and Daniela Silva from Centro de Materiais da Universidade do Porto (CEMUP) for her support with XPS spectra analyses.

Conflicts of Interest: The authors declare no conflict of interest.

\section{References}

1. Chen, J.; Wang, X.; Chen, T. Facile and green reduction of covalently PEGylated nanographene oxide via a 'water-only' route for high-efficiency photothermal therapy. Nanoscale Res. Lett. 2014, 9, 86. [CrossRef]

2. Tufano, I.; Vecchione, R.; Netti, P.A. Methods to Scale Down Graphene Oxide Size and Size Implication in Anti-cancer Applications. Front. Bioeng. Biotechnol. 2020, 8, 613280. [CrossRef]

3. Costa-Almeida, R.; Bogas, D.; Fernandes, J.R.; Timochenco, L.; Silva, F.; Meneses, J.; Goncalves, I.C.; Magalhaes, F.D.; Pinto, A.M. Near-Infrared Radiation-Based Mild Photohyperthermia Therapy of Non-Melanoma Skin Cancer with PEGylated Reduced Nanographene Oxide. Polymers (Basel) 2020, 12, 1840. [CrossRef] [PubMed]

4. Silva, M.; Alves, N.M.; Paiva, M.C. Graphene-polymer nanocomposites for biomedical applications. Polym. Adv. Technol. 2018, 29, 687-700. [CrossRef]

5. Mohammed, H.; Kumar, A.; Bekyarova, E.; Al-Hadeethi, Y.; Zhang, X.X.; Chen, M.G.; Ansari, M.S.; Cochis, A.; Rimondini, L. Antimicrobial Mechanisms and Effectiveness of Graphene and Graphene-Functionalized Biomaterials. A Scope Review. Front. Bioeng. Biotech. 2020, 8. [CrossRef] [PubMed]

6. Liu, S.B.; Zeng, T.H.; Hofmann, M.; Burcombe, E.; Wei, J.; Jiang, R.R.; Kong, J.; Chen, Y. Antibacterial Activity of Graphite, Graphite Oxide, Graphene Oxide, and Reduced Graphene Oxide: Membrane and Oxidative Stress. ACS Nano 2011, 5, 6971-6980. [CrossRef]

7. Hu, W.; Peng, C.; Luo, W.; Lv, M.; Li, X.; Li, D.; Huang, Q.; Fan, C. Graphene-based antibacterial paper. ACS Nano 2010, 4, 4317-4323. [CrossRef]

8. Fadeel, B.; Bussy, C.; Merino, S.; Vazquez, E.; Flahaut, E.; Mouchet, F.; Evariste, L.; Gauthier, L.; Koivisto, A.J.; Vogel, U.; et al. Safety Assessment of Graphene-Based Materials: Focus on Human Health and the Environment. ACS Nano 2018, 12, 10582-10620. [CrossRef] [PubMed]

9. Ou, L.L.; Song, B.; Liang, H.M.; Liu, J.; Feng, X.L.; Deng, B.; Sun, T.; Shao, L.Q. Toxicity of graphene-family nanoparticles: A general review of the origins and mechanisms. Part. Fibre Toxicol. 2016, 13. [CrossRef] [PubMed]

10. Liu, F.; Wang, C.; Sui, X.; Riaz, M.A.; Xu, M.; Wei, L.; Chen, Y. Synthesis of graphene materials by electrochemical exfoliation: Recent progress and future potential. Carbon Energy 2019, 12, 173-199. [CrossRef]

11. Farazas, A.; Mavropoulos, A.; Christofilos, D.; Tsiaoussis, I.; Tsipas, D. Ultrasound Assisted Green Synthesis and Characterization of Graphene Oxide. Int. J. Nanosci. Nanotechnol. 2018, 14, 11-17.

12. Méndez-Romero, U.A.; Pérez-García, S.A.; Fan, Q.; Wang, E.; Licea-Jiménez, L. Lateral size reduction of graphene oxide preserving its electronic properties and chemical functionality. RSC Adv. 2020, 10, 229432-229440. [CrossRef]

13. Marcano, D.C.; Kosynkin, D.V.; Berlin, J.M.; Sinitskii, A.; Sun, Z.; Slesarev, A.; Alemany, L.B.; Lu, W.; Tour, J.M. Improved synthesis of graphene oxide. ACS Nano 2010, 4, 4806-4814. [CrossRef] [PubMed]

14. Rodrigues, A.F.; Newman, L.; Lozano, N.; Mukherjee, S.P.; Fadeel, B.; Bussy, C.; Kostarelos, K. A blueprint for the synthesis and characterisation of thin graphene oxide with controlled lateral dimensions for biomedicine. 2D Mater. 2018, 5, 035020. [CrossRef]

15. Luo, J.; Cote, L.J.; Tung, V.C.; Tan, A.T.; Goins, P.E.; Wu, J.; Huang, J. Graphene oxide nanocolloids. J. Am. Chem. Soc. 2010, 132, 17667-17669. [CrossRef] [PubMed]

16. Şimşek, B.; Ultav, G.; Korucu, H.; Yartaşi, A. Improvement of the Graphene Oxide Dispersion Properties with the Use of TOPSIS Based Taguchi Application. Period. Polytech. Chem. Eng. 2018, 62, 323-335. [CrossRef]

17. Johnson, D.W.; Dobson, B.P.; Coleman, K.S. A manufacturing perspective on graphene dispersions. Curr. Opin. Colloid Interface Sci. 2015, 20, 367-382. [CrossRef]

18. Kim, J.; Kwon, S.; Cho, D.H.; Kang, B.; Kwon, H.; Kim, Y.; Park, S.O.; Jung, G.Y.; Shin, E.; Kim, W.G.; et al. Direct exfoliation and dispersion of two-dimensional materials in pure water via temperature control. Nat. Commun. 2015, 6, 8294. [CrossRef]

19. Li, D.; Muller, M.B.; Gilje, S.; Kaner, R.B.; Wallace, G.G. Processable aqueous dispersions of graphene nanosheets. Nat. Nanotechnol. 2008, 3, 101-105. [CrossRef] [PubMed]

20. Zhang, S.; Xiong, P.; Yang, X.; Wang, X. Novel PEG functionalized graphene nanosheets: Enhancement of dispersibility and thermal stability. Nanoscale 2011, 3, 2169-2174. [CrossRef] [PubMed]

21. Ain, Q.T.; Haq, S.H.; Alshammari, A.; Al-Mutlaq, M.A.; Anjum, M.N. The systemic effect of PEG-nGO-induced oxidative stress in vivo in a rodent model. Beilstein J. Nanotechnol. 2019, 10, 901-911. [CrossRef] 
22. Emiru, T.F.; Ayele, D.W. Controlled synthesis, characterization and reduction of graphene oxide: A convenient method for large scale production. Egypt. J. Basic Appl. Sci. 2017, 4, 74-79. [CrossRef]

23. Jasim, D.A.; Lozano, N.; Kostarelos, K. Synthesis of few-layered, high-purity graphene oxide sheets from different graphite sources for biology. 2D Mater. 2016, 3, 014006. [CrossRef]

24. Wojtoniszak, M.; Chen, X.; Kalenczuk, R.J.; Wajda, A.; Lapczuk, J.; Kurzewski, M.; Drozdzik, M.; Chu, P.K.; Borowiak-Palen, E. Synthesis, dispersion, and cytocompatibility of graphene oxide and reduced graphene oxide. Colloids Surf. B Biointerfaces 2012, 89, 79-85. [CrossRef]

25. Ganguly, A.; Sharma, S.; Papakonstantinou, P.; Hamilton, J. Probing the Thermal Deoxygenation of Graphene Oxide Using High-Resolution In Situ X-ray-Based Spectroscopies. J. Phys. Chem. C 2011, 115, 17009-17019. [CrossRef]

26. Araújo, M.P.; Soares, O.S.G.P.; Fernandes, A.J.S.; Pereira, M.F.R.; Freire, C. Tuning the surface chemistry of graphene flakes: New strategies for selective oxidation. RSC Adv. 2017, 7, 14290-14301. [CrossRef]

27. Some, S.; Kim, Y.; Yoon, Y.; Yoo, H.; Lee, S.; Park, Y.; Lee, H. High-Quality Reduced Graphene Oxide by a Dual-Function Chemical Reduction and Healing Process. Sci. Rep. 2013, 3. [CrossRef]

28. Wu, J.B.; Lin, M.L.; Cong, X.; Liu, H.N.; Tan, P.H. Raman spectroscopy of graphene-based materials and its applications in related devices. Chem. Soc. Rev. 2018, 47, 1822-1873. [CrossRef] [PubMed]

29. Muzyka, R.; Drewniak, S.; Pustelny, T.; Chrubasik, M.; Gryglewicz, G. Characterization of Graphite Oxide and Reduced Graphene Oxide Obtained from Different Graphite Precursors and Oxidized by Different Methods Using Raman Spectroscopy. Materials 2018, 11, 1050. [CrossRef] [PubMed] 